\title{
Report on MDA Workshop on Myotonic Dystrophy, 10 October 1994, Montreal, Quebec, Canada
}

At the annual meeting of the American Society of Human Genetics the Muscular Dystrophy Association held a half day workshop to discuss current findings and problems in myotonic dystrophy and to define future research directions. The workshop was organised by $\mathrm{Dr}$ Norine Stirpe (MDA) and Professor Be Wieringa (Department of Cell Biology and Histology, University of Nijmegen) and was attended by invited members of MDA funded research groups from USA, Canada, and Europe.

For those readers who have been on another planet for the past three years, myotonic dystrophy (DM) has been found to have an underlying trinucleotide repeat expansion, making it a member of the dynamic mutation class of disorders. The repeat, a $(\mathrm{CTG})_{\mathrm{n}}$, is unique among the dynamic mutations, being located in the $3^{\prime}$ untranslated region of a gene which encodes a protein kinase (DMPK). Understandably the meeting primarily focused on the effects of (CTG) $)_{n}$ expansion on the expression, properties, and localisation of DMPK protein. Possible involvement of other genes in the aetiology of the disease phenotype and progress towards cellular and animal models were discussed. It was clear to the audience that many new reagents, particularly antibodies and cDNA constructs, were available to investigate the normal cellular functions of DMPK and look for pathological changes in patient tissues.

Evidence supporting the multistep model proposed by Imbert et al (Nature Genet 1993; 4:72-6) for the population dynamics of the $(\mathrm{CTG})_{\mathrm{n}}$ repeat was presented by Steve Warren (Emory University) from studies of African, Asian, and native American populations. The model predicts a premutation allele of $n=20$ or more repeats, which from haplotype data arose from expansion of the most frequent $(\mathrm{CTG})_{5}$ allele. Under this model the repeat number would continue to expand until a threshold of $n=40-50$ repeats was crossed when the $(\mathrm{CTG})_{\mathrm{n}}$ would become unstable on transmission through the germline leading to larger, DM causing expansions. Warren's findings suggest that the intermediate sized alleles (CTG) ${ }_{11-17}$ are stable from studies of Navajo Indians as no alleles of more than (CTG) ${ }_{13}$ are found in this population. Further support for the multistep model comes from the very low frequency of $\mathrm{DM}$ in indigenous African populations. Disease frequency correlates with the frequency of alleles in the (CTG) ${ }_{19-30}$ range in the study groups. Part of the haplotype associated with DM alleles includes an insertion/deletion event $5 \mathrm{~kb}$ distal to the $(\mathrm{CTG})_{\mathrm{n}}$. In African populations there is discordance between the insertion/deletion polymorphism and the repeat expansion, suggesting that progression of the repeat number to larger ranges has occurred more than once in human history.

Further support for the role of segregation distortion (possibly meiotic drive) in maintaining DM alleles in the population, despite the severe reduction of reproductive fitness in the congenital cases, was presented by Helen Harley (University of Wales Medical School). The analysis presented differed from that in a previously published report (Carey et al, Nature Genet $1994 ; 6: 117-18$ ) in that only small or large allele size classes were used. These differences in methods of analysis might account for the differences reported in the two studies. The Cardiff group found that there was significant transmission distortion from mothers to offspring for the larger alleles. The previous study and a recently published report (Gennarelli et al, $\mathcal{F}$ Med Genet 1994;31:980) showed a similar segregation distortion effect in male transmission. It is possible that combining the data sets from these different studies will show that the distortion is significant in both sexes and determine whether the effect is entirely repeat driven or the result of surrounding haplotype effects.

Darren Monckton (Baylor College of Medicine) presented small pool PCR data showing a strong trend towards (CTG) $)_{n}$ amplification in sperm, with fewer than $1 \%$ of sperm having smaller repeat alleles than the progenitor. $\mathrm{He}$ also presented data showing progressive enlargement of repeat size over time in the white blood cells of patients. The sperm data showed a mean increase of +180 repeats within a normal distribution of allele sizes from a male with 75 repeats in his leucocyte DNA. Tetsuo Ashizawa (Baylor College of Medicine) presented further data showing the ongoing instability of the repeat expansion after birth in eight monozygotic twin pairs. In five of the pairs the repeat size was concordant in lymphocyte DNA. In one of the three non-concordant pairs the difference was pronounced, although the phenotype was concordant in all pairs. As had been previously reported, the expansion sizes were markedly larger in muscle compared to 
leucocyte DNA from the same patient. Claudine Junien (INSERM Unite 383) suggested that the discordant twins might result from diamniotic pairs. She presented data suggesting that where the parental repeat expansion is small $(<0.35 \mathrm{~kb})$ there is an excess of males giving rise to larger expansions, suggesting a sex specific sensitivity to repeat length in the expansion mechanism. Keith Johnson (Charing Cross and Westminster Medical School) confirmed that his group had also seen continuation of the expansion over time in lymphocytes from adult patients.

Johnson discussed the factors that were being considered in designing a preimplantation diagnosis protocol for DM. The crux of the problem is that the large expansions can only be detected by Southern blots and only PCR based methods can be applied to single cells. The protocol being developed by Johnson and coworkers at the Royal Postgraduate Medical School (London) involves the use of a highly informative linked marker (X75) in addition to the (CTG) $)_{n}$ PCR and a preamplification step that overcomes using multiplex approaches, developed by Norman Arnheim's group (University of Southern California).

Richard Moxley (Rochester) presented work from his group investigating the possible direct link between the insulin resistance shown by DM patients and the DM $(C T G)_{n}$. It is possible that DMPK directly phosphorylates the insulin receptor, since it is known to be phosphorylation sensitive or that DMPK lies in a pathway downstream of the receptor. Moxley described a new clinical disorder, inherited as an autosomal dominant trait, with no (CTG) expansion and which is not linked to chromosome 19 markers. The disorder has clinical similarities to DM, with cataract and myotonia associated with muscle weakness. However, the distribution of muscle wasting and weakness is markedly different from DM, being proximal rather than distal. It is marked more by muscle pain than atrophy with sparing of the muscles of the face. The new condition has been termed proximal myotonic myopathy (PROMM). In addition to excluding linkage to chromosome 19 markers, the sodium channel locus on chromosome 17 and the chloride channel on chromosome 7 have also been excluded as candidate loci in a subset of families affected by PROMM.

It has been speculated that ion channels, which are known to harbour mutations in patients with some myotonic conditions, are potential substrates for DMPK. Randall Moorman (University of Virginia) coinjected mRNAs of the muscle sodium channel and DMPK containing either (CTG $)_{5}$ or (CTG) ${ }_{75}$ into Xenopus oocytes. The (CTG) ${ }_{5}$ mRNA led to a reduced current amplitude being generated from the channel indicating that phosphorylation of the sodium channel by DMPK may have a regulatory role on channel function. By site directed mutagenesis of a known phosphorylation site in the channel, the effect of DMPK co-expression on current amplitude was abolished. The presence of the (CTG) ${ }_{75}$ mRNA did not affect the ability of the (CTG) mRNA to modulate channel function, but by itself failed to produce as marked a decrease in current amplitude as the (CTG) ${ }_{5}$ mRNA. Another potential target protein, phospholammin, showed similar effects with the (CTG) $)_{5}$ and $(\mathrm{CTG})_{75}$ repeat species.

Luba Timchenko (Baylor College of Medicine) used northern analysis along with antipeptide and antifusion protein antibodies to investigate steady state levels of DMPK mRNA and protein in transformed lymphoblasts and fibroblasts from a homozygote with alleles of $(\mathrm{CTG})_{330}$ and (CTG) ${ }_{700}$. Only weak bands of $7 \cdot 1 \mathrm{~kb}$ (estimated to be less than $33 \%$ of normal intensity) were seen on northern blots. The antipeptide antibody detected a protein species of $55 \mathrm{kDa}$ and the antifusion protein antibody bands of $55 \mathrm{kDa}$ and $72 \mathrm{kDa}$. The $72 \mathrm{kDa}$ species was estimated to be present at a two to three fold reduction compared to normals. Only the $72 \mathrm{kDa}$ protein was found in the phosphorylated form in fibroblasts. In subcellular fractions the $72 \mathrm{kDa}$ protein was cytoplasmically localised and a nuclear localisation was observed for the $55 \mathrm{kDa}$ species. The $72 \mathrm{kDa}$ protein has kinase activity, autophosphorylates and phosphorylates synthetic substrates containing serine target sites. The $72 \mathrm{kDa}$ species was also able to phosphorylate the dihydropyridine receptor in vitro.

Robert Korneluk (Children's Hospital of Eastern Ontario) presented results obtained with antibodies raised against a fusion protein corresponding to exons $11-15$ of the DMPK gene. These antibodies detect $72 \mathrm{kDa}$ and $84 \mathrm{kDa}$ species on western blots of DM tissues. Fluorescent immunocytochemistry detected DMPK protein at the neuromuscular junction of skeletal muscle, intercalated discs in cardiac muscle, and in the choroid plexus of the brain. Immunoprecipitated DMPK protein showed kinase activity on histone substrate.

Michael Siciliano (Anderson Cancer Center) reported on allele specific transcription analysis using expressed RFLPs in patient mRNA. Primary versus processed transcripts originating from the normal and DM alleles were investigated in patient and control muscle tissue and cell lines. Ten patients were informative for the primary transcript analysis and three for the processed analysis; two were informative for both. Primary transcript was detected from both alleles in skeletal muscle and cell lines from DM patients but there was reduced processed transcript derived from the expanded allele when steady state levels of mRNA were examined. This effect was not seen when transcripts from a neighbouring gene (N9) were studied in a similar fashion. The mechanism by which the mature transcript decreases was investigated and shown not to be the result of gross changes in mRNA stability. Overall there were no changes in locus activity for the expanded DM allele compared to the control nor were there detectable shifts in abundance of different splice variants of DMPK.

Vickie Funanage (Alfred I DuPont Institute) reported on transcriptional studies of chromosomes from patients segregated in somatic cell hybrids. RTPCR on mature mRNA failed to detect a transcript in the hybrid cells con- 
taining the chromosome bearing the expanded repeat allele. N9 was normally expressed from both alleles in this system. Junien reported a similar lack of mature mRNA from DMPK in tissues from a congenital DM case. In this case the total RNA from DMPK was increased above control levels suggestive of upregulation of the normal allele, although in tissues from a 20 week congenital DM fetus there was less DMPK transcript than in controls. The RTPCR results depended on where the primers were located in the DMPK transcript. The congenital child samples gave 4 to 10 fold higher levels of DMPK mRNA with primers at the $3^{\prime}$ end and seemed to be even higher with primers specific for the $5^{\prime}$ end. This suggested that there might be truncation of the transcripts because of the repeats.

David Brook (University of Nottingham) presented an analysis of the genomic region containing the (CTG) $)_{n}$ repeat, which is located in a $\mathrm{CpG}$ island. $\mathrm{CpG}$ islands are usually located at the $5^{\prime}$ end of genes. The $(C T G)_{n}$ is at the $3^{\prime}$ end of DMPK. These observations suggest that the (CTG) $)_{n}$ expansion could be affecting the expression of genes downstream of DMPK. Several exons of between 100 and $150 \mathrm{bp}$ in length have been isolated using exon trapping from the region within $10 \mathrm{~kb}$ downstream of the repeat. When used as probes, these exons detect conserved sequences on zoo blots suggesting they are parts of transcripts, but no corresponding cDNAs have been isolated to date. An exon $50 \mathrm{~kb}$ downstream has been used to isolate two cDNAs which are part of a $3 \mathrm{~kb}$ mRNA transcript with weak homology to transcription factors. This gene is intronless. A gene upstream of DMPK, 59 (N9 in the mouse), has also been characterised. In DM fibroblasts Brook sees no alterations in level of expression of DMPK or 59.

Studies of gene expression from the mouse syntenic region were presented by $\mathrm{Be}$ Wieringa. The mouse homologue of $59, \mathrm{~N} 9$, has five exons and a clean initiation site for transcription. However, the N9 polyadenylation signal is weak and the transcription start site of DMPK is very near to it. N9 and 59 proteins have $95 \%$ similarity, are encoded by a gene spanning only $8 \mathrm{~kb}$ of both mouse and human genomes, and has no significant homologies to proteins in the database. N9 expression was investigated through mouse development using whole embryo in situ hybridisation. This showed embryonic and not placental expression with the highest signals coming from forebrain and retina during early development. Later in development expression was found in the retina, CNS, and olfactory bulb. In adult animals expression was found in some patches of epithelium in seminiferous tubules, suggesting that this might correlate with areas of active spermatogenesis.

Robert Korneluk presented results from in vitro expression studies of the DMPK promoter using a construct extending $700 \mathrm{bp}$ upstream of the initiator ATG and extending $3^{\prime}$ into the exonic sequences of DMPK. The first intron of DMPK was found to contain three $\mathrm{E}$ boxes, which are conserved muscle specific tran- scription factor binding sites which are orientation specific and enhance expression of the constructs.

Peter Strong (Royal Postgraduate Medical School) presented results obtained using antibodies raised against peptides within the kinase domain of DMPK. A $52 \mathrm{kDa}$ band was predominantly recognised on western blots but in some tissues a $70 \mathrm{kDa}$ band was also seen. The $52 \mathrm{kDa}$ protein band is being sequenced to determine its relationship to DMPK gene products. Fibre type differences in the staining patterns with this antibody were seen, with greater signal intensity in slow muscles. Immunofluorescence studies suggested a perinuclear localisation with some additional staining of Golgi in $\mathrm{C} 2$ cells. In mature muscle biopsies the staining is speckled and diffuse. No qualitative or quantitative differences in this protein species were seen between control and congenital DM muscle biopsies on western blots.

Patricia Groenen (University of Nijmegen) expressed cDNA constructs representing various human and mouse DMPK isoforms with incomplete kinase domains in COS cells to produce immunogen. In addition, a peptide specific to the product of exon 8 of DMPK was used to produce antibodies which detected expression throughout the cytoplasm by immunofluorescence. The antibodies against variants IV and V (nomenclature as in Jansen et al, Nature Genet 1992;1:261-6) stained perinuclear structures (probably Golgi and endoplasmic reticulum). These results suggest that different isoforms are routed differently in the cell and that the C-terminus of the protein determines this.

Be Wieringa showed in situ data for DMPK in mouse tissues. At embryonic day 10.5 the expression is mainly in the myotomes of the developing somites. Later it is expressed more widely in smooth and skeletal muscle. Very high levels of expression are seen in the developing tongue and oesophagus. Expression occurs in cardiac tissues at embryonic day 16 and becomes maximal at day 18. DMPK is also expressed in the ductus deferens but not in the testis itself. In the ovary there is some expression in the gonadal tissue as well as the smooth muscle of the ovarian duct. Expression is only seen in the brain after birth where it is most evident in the granular cell layer of the cerebellum. The expression is abolished in knockout mice (see later).

Henry Epstein (Baylor College of Medicine) investigated the enzymatic activity of expressed protein from various cDNA constructs. DMPK shows a preference for serine over threonine and requires higher levels of inhibitors than classical kinases, indicating that it probably represents a new class. DMPK protein is highly expressed in lens tissue in a region consistent with a role in cataract formation (an early symptom of the disease). The results of peptide mapping of the recombinant protein used to raise the antibodies were very similar to those generated by the immunoprecipitated protein from heart. The lens protein had similar peptide fragments at high molecular weight to the re- 
combinant protein. The polyclonal antibodies raised against the recombinant protein reacted with the cuboidal epithelium from which the lens arises. In muscle the antibody only reacts with a subset of fibres. There was also evidence for different phosphorylation states and possible isoform variation. Phospholamban was shown to be phosphorylated by the immunoprecipitated DMPK, but this may represent a relatively non-specific substrate for protein kinases.

Jack Puymirat (CU Laval Research Centre) presented studies of the $54 \mathrm{kDa}$ protein species identified by an antipeptide antibody. The immunoprecipitated protein preferentially phosphorylated tyrosine residues in skeletal muscle, and serine residues in heart. Switching of substrate specificity in a tissue specific fashion appeared to be dependent on the phosphorylation state of DMPK. Ben Perryman (University of Colorado) used an antibody generated in Robert Korneluk's laboratory against a fusion protein specific to exons 11-15 of the DMPK sequence. Two prominent protein bands were detected on western blots at around $69 \mathrm{kDa}$ in a range of tissues and COS cells. This antibody specifically reacts against intercalated discs in rat and mouse cardiac muscle and the neuromuscular junction (NMJ) in skeletal muscle of these species. The NMJ staining in human tissues is not as clear cut with this antibody as it is in mouse tissues. The antibodies were used to purify DMPK and evidence was presented that there was an inhibitory subunit from reconstitution studies. Epstein commented that this putative inhibitory subunit was in the membrane fraction.

Be Wieringa summarised his group's progress towards the development of a mouse model of DM. This involved overexpression studies in transgenic animals and knockouts using gene targeting. Exon 7 had been replaced in the targeting construct and heterozygous and homozygous targeted offspring had been born. These mice reproduce normally and have no phenotypic manifestations of DM. No histological changes were seen in their tissues compared to wild type. The overexpression constructs led to up to $50 \%$ higher expression of human DMPK protein in skeletal muscle of the transgenic offspring. The tissue specificity of expression was correct and corresponded to the sites of expression of the mouse DMPK homologue. In cardiac tissues of these animals some patchy myocyte disarray was seen, histologically similar to hypertrophic cardiomyopathy. These animals could not be bred to homozygosity.

A summary discussion took place led by $\mathrm{Be}$
Wieringa, which concluded that the antibody results are still confusing. The evidence points to the $70 \mathrm{kDa}$ protein product of the DMPK gene being the key species rather than the 52 or $54 \mathrm{kDa}$ species detected by the antipeptide antibodies. It is likely that the lower molecular weight species are detected through cross reactivity with abundant kinases present in muscle and other tissues. This is supported by the fact that antibodies raised against the expressed C-terminal half of DMPK only detect the larger species. This issue will only be unequivocally answered when the $52 / 54 \mathrm{kDa}$ protein species has been sequenced and its relationship, if any, to the DMPK gene determined. While both over and under expression mouse models have been developed, neither produce the DM phenotype. It was commented by Kurt Fischbeck (Philadelphia) that while the evidence was still patchy, the tissue distribution of DMPK still pointed to it being strongly implicated in the aetiology of DM.

In conclusion, we now know more about the properties and potential substrates of DMPK, but we still have no hard data concerning changes in its properties, localisation, or abundance that implicate it in the aetiology of the disease symptoms. The gene knockouts and overexpressing transgenic animals do not produce models of $\mathrm{DM}$, indicating that the genetic mechanism is unlikely to be a straightforward loss or gain of expression of DMPK. However, these mice will be invaluable in determining the function of DMPK. Our genetic models based on population samples support a multistep process leading to expansion of the repeat from a founder mutation. The segregation distortion data predict that the prevalence of premutation alleles in the population will increase over time, making it all the more pressing that the plethora of mechanistic questions posed by DM are answered in a timely fashion.

KEITH J JOHNSON* Genetics Unit, Department of Anatomy, Charing Cross and Westminster Medical School, Fulham Palace Road, London W6 8RF, UK

MICHAEL J SICILIANO Department of Molucular Genetics, University of Texas Anderson Cancer Center, 1515 Holcombe,

Houston, TX 77030, USA

*Present address: Genetics Laboratory, IBLS, Pontecorvo Building, University of Glasgow, Church Street, Glasgow G11 5JS, UK. 